\title{
Technical advancements and protocol optimization of diffusion-weighted imaging (DWI) in liver
}

Ping Ni, Yuning Lin, Qun Zhong, Ziqian Chen, Kumar Sandrasegaran, Chen Lin

\begin{abstract}
An area of rapid advancement in abdominal MRI is diffusion-weighted imaging (DWI). By measuring diffusion properties of water molecules, DWI is capable of non-invasively probing tissue properties and physiology at cellular and macromolecular level. The integration of DWI as part of abdominal MRI exam allows better lesion characterization and therefore more accurate initial diagnosis and treatment monitoring. One of the most technical challenging, but also most useful abdominal DWI applications is in liver and therefore requires special attention and careful optimization. In this article, the latest technical developments of DWI and its liver applications are reviewed with the explanations of the technical principles, recommendations of the imaging parameters, and examples of clinical applications. More advanced DWI techniques, including Intra-Voxel Incoherent Motion (IVIM) diffusion imaging, anomalous diffusion imaging, and Diffusion Kurtosis Imaging (DKI) are discussed.
\end{abstract}

\section{Keywords}

Liver diffusion weighted imaging, Intra-voxel incoherent motion diffusion, Anomalous diffusion, Diffusion kurtosis

\section{Introduction}

Diffusion-weighted imaging (DWI) is a noninvasive imaging technique sensitive to the mobility of water molecule at cellular and macromolecular level, much smaller than the scale of the spatial resolution or voxel size of the images [1,2]. At such level, the motion of water is strongly influenced by the inter-cellular and intra-cellular environment which changes with tissue type and pathology. For example, for tissue or tumor with high cellularity, the movement of water molecule is more restricted because of narrower inter-cellular space. In addition, the diffusion weighted MRI

This is the author's manuscript of the article published in final edited form as:

Ni, P., Lin, Y., Zhong, Q., Chen, Z., Sandrasegaran, K., \& Lin, C. (2016). Technical advancements and protocol optimization of diffusion-weighted imaging (DWI) in liver. Abdominal Radiology, 41(1), 189-202.

http://doi.org/10.1007/s00261-015-0602-x 
signal is sensitive to transport process such as the blood flow in the capillary vessels. The ability to probe tissue microstructure makes DWI a powerful tool in clinical diagnosis of abdominal diseases and differentiation of different types of lesions. In last few years, DWI has become of routine part of abdominal MRI [3].

DWI has been found to be sensitive in detecting a variety of focal liver lesions including cholangiocarcinoma [4], inflammatory myofibroblastic tumor (IMT) [5], intrahepatic bile duct adenoma (BDA) [2], hepatocellular carcinoma (HCC) [6-8], hemangioma [9], focal nodular hyperplasia (FNH) [10-12], and simple hepatic cyst [13]. For the detection of focal liver lesions, DWI performs better than $\mathrm{T} 2$ weighted sequences but is generally inferior to $\mathrm{T} 1$ weighted dynamic gadolinium enhanced (DCE) images [3, 14-16]. Therefore, DWI can be complementary to standard MR protocol for imaging focal liver lesion, especially for patients who has renal dysfunction or other contraindications to gadolinium based contrast agents.

The liver is the most common abdominal organ of metastases and liver metastases is the most frequently encountered malignant liver lesions [17]. While DWI cannot replace gadolinium enhanced sequences $[18,19]$, it has been found to be beneficial in many ways. Combining DWMRI with Gadolinium-EOB-DTPA-enhanced series significantly improved the detection of colorectal liver metastases [20, 21]. However, DWI has been reported as being incapable of differentiating malignant from benign hepatic solid lesions [22-24]. Although the addition of DWI sequences to Gd-EOB-DTPA enhanced MRI may not significantly increase diagnostic accuracy, the increase in diagnostic confidence would justify the DWI sequences in a dedicated MRI protocol [25]. For instance, appearances of hypointensity on gadoxetic acid-enhanced hepatobiliary phase images and hyperintensity on high-b-value DWI may indicate HCC rather than regenerative or dysplastic nodules in cirrhotic liver [26].

DWI can also serve as a biomarker for treatment response of liver tumor [27, 28]. Several studies have reported that the post-trans-arterial chemoembolization (TACE) or post-radiofrequency ablation (RFA) ADC values of hepatic malignancies were significantly higher than baseline values $[29,30]$. With good response to treatment, tumors can exhibit a decline of signal intensity at high b-value images or an increase in ADC value, which may be secondary to treatment-induced cystic or necrotic changes and cell death [31]. 
Several studies have demonstrated the capability of DWI in early detection and classification of liver fibrosis and fatty liver diseases [32-37]. The common theory for studying liver fibrosis by DWI is that the interstitial space of fibrotic liver parenchyma may be narrower than normal, which is accompanied by restricted diffusion and declined ADC values. A relationship between the degree of fibrosis and ADC value was observed. However, using DWI for evaluating liver fibrosis still remains controversial [37-39]. An inverse relationship between ADC and hepatic fat fraction was documented, although other MRI techniques are better suited for this purpose [40, 41]. Conventional DWI was also found helpful in diagnosing parenchymal disorders post liver transplant as significant lower ADC was found in patients with at least one parenchymal disorder [42].

Driven by its increasing clinical applications, there have been significant technical advancements in both diffusion-weighted image acquisition and post-processing recently. Such effort has not only dramatically improved the image quality of liver DWI, but also offers addition information beyond conventional diffusion-weighted images and apparent diffusion constant (ADC) maps. The purpose of this article is to provide a comprehensive review of the latest progress in liver DWI with emphasis on how conventional DWI should be optimized and performed based results from recent studies and what new possibilities beyond conventional DWI are made possible by the exciting technical advancements. For conventional liver DWI, a set of optimized basic imaging parameters is provided. Along with the analysis of the trade-offs with different imaging options, it may server as a reference for clinical implementation. For advanced liver DWI, the remaining challenges are discussed so they can be taken into consideration for clinical translation.

\section{Conventional Diffusion-weighted Imaging and Applications in Liver}

\section{Conventional Diffusion-weighted Imaging (DWI) Technique}

By acquiring images with two or more diffusion weightings and fitting the signal change with a mono-exponential decay function, the so called apparent diffusion coefficient (ADC) can be estimated. Because of technical challenges, hepatic DWI started around 2005, much later than its application in the brain. DWI for liver imaging was reviewed separately by Taouli et al and Kele et al in 2010 [43, 44]. This article will focus on the technical developments and the new applications since then. 


\section{Diffusion-weighted Echo-planar Imaging (EPI)}

In order to make MRI signal sensitive to the diffusive motion of water molecules, a diffusion encoding module can be added to a MRI pulse sequence. A simple diffusion encoding module consists of two large gradient pulses of equal size separated by an $180^{\circ}$ refocusing radio frequency (RF) pulse as shown in figure 1.

The first gradient pulse before the $180^{\circ} \mathrm{RF}$ pulse introduces a phase shift which depends on the relative spatial location of the water molecule in the direction of the magnetic field gradient. For static water proton, such phase shift is fully compensated and recovered by the second gradient pulse of equal size after the $180^{\circ}$ refocusing RF pulse. For water molecules that move in the direction of the magnetic field gradient during the time interval of those two gradient pulses, the phase shift produced by the first gradient may not be fully undone by the second gradient pulses. A net phase shift is therefore created by such diffusion sensitizing module. For a large number of diffusing water molecules undergoing random Brownian motion, their displacements along the diffusion sensitizing direction would be a Gaussian distribution and the sum of their phase shifts would be zero. However, the net magnetization, which is the sum of magnetic moments from water protons, will be reduced due to their phase differences. The change of magnetization and, consequently, MRI signal intensity depends on the diffusivity, or apparent diffusion coefficient (ADC), and the amount of diffusion weighting which is quantified as b-value in the following equation [45].

$$
\mathbf{S} / \mathbf{S}_{b=0}=e^{-b^{*} \mathrm{ADC}}
$$

where $b=\gamma^{2} G^{2} \delta^{2}(\Delta-\delta / 3)$.

While many acquisition techniques may be used to acquire the diffusion-weighted or encoded signal, a fast and efficient acquisition insensitive to bulk motion such as single-shot technique would be beneficial. This is because the diffusion sensitizing module itself is relatively long since it takes time to play out the large diffusion sensitizing gradient pulses especially when high bvalues are needed and maximum gradient amplitude can be generated by scanner hardware is limited. It is therefore desirable to acquire all the data needed to reconstruct the diffusion-weighted image after a single excitation and diffusion sensitizing instead of repeating the diffusion sensitizing multiple times, one for each phase encoding steps. Single-shot Echo Planar Imaging 
(SS-EPI) is a sequence that meets such requirements. Figure 2 shows the sequence diagram of diffusion-weighted SS-EPI which diffusion sensitizing in all three orthogonal directions.

While the water diffusion in the tissue of abdominal and pelvis organs is believed to be mostly isotropic, i.e. $\mathrm{D}_{\mathrm{xx}}=\mathrm{D}_{\mathrm{yy}}=\mathrm{D}_{\mathrm{zz}}$, averaging the diffusion weighting from all three directions, $\left(\mathrm{D}_{\mathrm{xx}}+\right.$ $\left.\mathrm{D}_{\mathrm{yy}}+\mathrm{D}_{\mathrm{zz}}\right) / 3$, is still beneficial and allows the results to be more consistent and less direction dependent. As shown in Figure 2, diffusion sensitizing can be applied in three orthogonal directions at the same time without increasing the length of diffusion sensitizing module.

\section{$\underline{\text { Acceleration of DWI acquisition }}$}

Like almost any other imaging technique, a fast DWI acquisition is always desirable [46]. With the recent advances in phase array coil hardware and reconstruction algorithms, parallel imaging is used routinely to accelerate DWI acquisition. Using parallel imaging in diffusion-weighted SSEPI has addition advantages beyond reducing the number of k-space lines or the EPI echo train length in this case. Because, by reducing the echo train length in DW-SS-EPI sequence, the minimal echo time (TE) can also be reduced. A shorter TE also means less T2 decay or a boost in signal-to-noise ratio (SNR), which partially offsets the loss of SNR from parallel imaging acceleration $[46,47]$. Another advantage with shorter echo train length through parallel imaging is less geometric distortion. This is important for abdominal DWI as magnetic field inhomogeneity is often found at the boundaries of tissue and air due to susceptibility effect. As explained later, parallel imaging is also an effective technique for reducing susceptibility artifact. Therefore, it is important to take advantage of parallel image if available in DWI protocol in order to achieve better results.

Another promising technique for the acceleration of DWI is through simultaneous multi-slice excitation or slice acceleration [48]. In simultaneous multi-slice excitation, a multiband radiofrequency (RF) pulse excites multiple 2D slices at the same time and the signals from simultaneous acquired slices are separated based on the coil sensitivity profile difference in the slice direction. Unlike parallel imaging, there can be little or no SNR penalty with slice acceleration. In fact, slice acceleration and parallel image can be combined to achieve even higher acceleration factor if the design of multiple channel phased array coil supports acceleration in both slice and phase encoding directions. The initial application of slice accelerated DWI in liver has produced encouraging 
results where comparable image quality and $\mathrm{ADC}$ values were obtained from both conventional DWI and slice accelerated DWI with an acceleration factor of $2[49,50]$.

\section{$\underline{\text { Diffusion weighting with tetrahedral gradients }}$}

In a conventional DWI sequence, orthogonal $\mathrm{x}, \mathrm{y}$, and $\mathrm{z}$ diffusion gradient pulses are applied separately, and isotropic fusion weighted images are obtained by averaging three images with orthogonal diffusion sensitizing gradients. However, the orthogonal $\mathrm{x}, \mathrm{y}$, and $\mathrm{z}$ diffusion gradients pulses may be simultaneously applied as shown in figure 3, which shortens the duration of diffusion gradient pulses and consequently the TE for the same b-value. Isotropic diffusionweighted images can be obtained by using four different tetrahedral vector images [51].

\section{Motion compensation}

Although DW-SS-EPI itself is one of fastest acquisition techniques, motion compensation is necessary for certain abdominal applications of DWI such as liver for two main reasons. First of all, the calculated of ADC requires at least two diffusion-weighted images with different b-values. If there is any mis-registration between the two images, there would be an error in the calculated ADC map. Secondly, the bulk motion of the tissue during diffusion sensitizing cannot be distinguished from actual diffusive motion and therefore causes a bias in the measured ADC. To avoid such problem caused by respiratory motion, liver DWI may be acquired with breath-hold $(\mathrm{BH})$, respiratory-triggered $(\mathrm{RT})$ or free-breathing $(\mathrm{FB})$ with multiple averages.

DWI acquisition with breath-hold is quicker than with respiratory-triggered or free-breathing. The suspension of respiratory motion and the short acquisition time reduces artifacts due to macroscopic physiological motions (respiration, peristalsis). On the other hand, breath-hold is less reproducible [52] and one may need to make certain compromises in anatomical coverage due to limited number of slices, spatial resolution due to shorter EPI echo train length and signal-to-noise ratio (SNR) due to shorter TR in order to fit the entire acquisition in one or multiple breath-holds.

Respiratory-triggered DWI acquisition requires synchronization with patients' respiratory cycle which can be monitored using a "respiratory" belt or bellow placed around or on the patient's abdomen or with a navigator technique, such as prospective acquisition correction technique (PACE) [53], to track the position of patient diaphragm [54]. The acquisition takes place at the 
end of expiration when motion is minimal. Respiratory-triggered sequences allow for high quality images in patients who have difficulty with holding breath, but takes longer scan time. Typical acquisition times are 3-6 minutes [43]. Kandpal et al found respiratory-triggered DWI provides better image quality and SNR than breath-hold DWI for the evaluation of focal liver lesions without any compromise in the calculated ADC values [55]. Despite its advantages, respiratorytriggered DWI may suffer from pseudo-anisotropy artifact which originates from the complicated local hepatic movements, such as extension, contraction and rotation, and causes errors in ADC measurement [56].

Free-breathing DWI acquisition relies on multiple excitations, which may be necessary anyway to achieve adequate SNR, to average out the effect since acquisitions with different $b$-values or repeated acquisition with the same b-value is likely to be from different parts of the respiratory cycle [57]. While free-breathing acquisition is time efficient, the image maybe blurred from the averaging and the ADC value maybe elevated due to bulk motion [58].

Several studies have compared image quality and ADC reproducibility between motion compensation techniques. Sandberg et al and Kandpal et al both compared respiratory triggered and breath-hold sequences and found better image quality with respiratory triggering [55, 59]. Taouli et al evaluated the usefulness of navigator echo triggered diffusion acquisition. They also found improvement in image quality and ADC measurement compared with standard breath-hold sequences at a cost of longer acquisition time [54]. In contrast, Kwee et al found higher ADC values with triggered acquisition [60]. Nasu et al attributed the inaccuracy of ADC measurement with respiratory triggering to pseudo-hepatic anisotropy artifact, predominately in the left lobe [56]. Such artifact maybe compensated using a larger number of directions in diffusion encoding [61]. Nasu et al also compared DWI acquired with free-breathing versus respiratory triggering; higher accuracy of ADC measurement was reported using respiratory triggering [62]. Naganawa et al compared $\mathrm{ADC}$ values obtained with respiratory triggering and free-breathing [63]. They found higher $\mathrm{ADC}$ values in the right lobe with free-breathing, suggesting that $\mathrm{ADC}$ values are influenced by respiratory motions. They also found that ADC values in the left lobe are about the same in both respiratory triggering and free-breathing, suggesting that ADC in the left lobe is mostly influenced by cardiac motion. 
To reduce impact of cardiac motion, Bruegel et al suggested using pulse triggering [64]. Murtz et al also analyzed the influence of cardiac motions on diffusion measurement and found that ADC values without cardiac triggering were artificially higher than those obtained with cardiac triggering [65]. However, simultaneous use of both respiratory and cardiac triggering makes the DWI acquisition extremely long; thus Nasu et al suggested using only respiratory triggering, but with more averages to increase the percentage of data acquisition during diastole [56]. Liau et al also proposed an improved averaging method to mitigate cardiac motion artifacts in repeat measurements [66].

Other methods for compensation motion artifacts include the use of velocity compensated diffusion gradients [51]. Using dual bipolar gradient waveform, velocity compensated diffusion gradients remove all phase sensitivity to constant velocity motion (first-moment) during the diffusion-weighting period. The drawbacks of using velocity compensated diffusion gradients is that it's longer that the conventional mono-polar gradients and it does not compensate for higher than first moments (e.g. acceleration).

\section{$\underline{\text { Artifact Reduction }}$}

While SS-EPI sequence provides efficient image acquisition for DWI, it's notoriously prone to artifacts. The commonly seen artifacts include: chemical shift artifacts, B0 inhomogeneity artifacts and Nyquist ghosting artifacts as illustrated in figure 4.

In EPI sequence, the chemical shift of fat resonance causes a large displacement of fat signal along the phase encoding direction. If the fat signal is not completely suppressed, it may super impose on the region of interest. Therefore, for DW-EPI in abdomen where fatty tissue is extensively present, reliable fat suppression is necessary [1]. Recently, more advanced fat suppression techniques based on inversion recovery such as Spectral Pre-saturation with Inversion Recovery [SPIR] and SPectral Attenuated Inversion Recovery [SPAIR] have been introduced and integrated in DW-EPI. To further improve fat suppression, a new approach of combining Dixon/IDEAL with DW-EPI has been attempted and yielded promising results [67,68]. Lee et al also compared a fat suppression technique based on slice-selection gradient reversal [SSGR] with SPIR fat suppression at 3T and found SSGR was significantly better than SPIR for liver DWI [69]. 
Based on a long gradient echo train, the EPI sequence is inherently sensitivity to B0 inhomogeneity or magnetic susceptibility artifacts caused by tissue and air interface in the abdominal region, especially at higher field such as $3 \mathrm{~T}[3,43]$. The most effective way to minimize such artifact is by reducing the echo train length in EPI. One option is using partial Fourier and parallel imaging to reduce the number of phase encoding steps or echoes in EPI as discussed previously. Another option is to use high receiver bandwidth and consequently high gradient amplitude to reduce the echo spacing. However, with the increase of receiver bandwidth, the SNR will also decrease. Therefore, there is a point of diminish return in echo spacing reduction with high receiver bandwidth which is scanner hardware dependent. Other than optimizing acquisition parameters, one can also reduce the inhomogeneity by improving B0 shim. One of the strategies is to optimize the shim one slice at a time (iShim) instead of the entire imaging volume [70].

While the mono-polar diffusion sensitizing scheme has the simplest gradient waveform and the shortest TE, it's also less favorable in terms of compensating for eddy current. More sophisticated diffusion sensitizing schemes use gradient pulses of different polarity to cancel the eddy currents and reduce the artifact. However, because other contributions such as B0 inhomogeneity are more prominent in liver application, the improvement from eddy current reduction may not be as appreciable and worthy of the increase in both TE and scan time.

\section{Protocols optimization}

In order to produce high quality ADC map and accurately quantify ADC for regions for interest, the first step is to obtain high quality diffusion-weighted images with good SNR and free of artifacts. Based on the previous discussions, an optimized DW-SS-EPI for liver should employ a robust fat suppression technique to reduce the chemical shift artifact, a combination of parallel imaging, partial Fourier and reasonable high receiver bandwidth to minimize the TE and distortion

artifact and an appropriate scheme to compensate for respiratory motion and cardiac motion if necessary. For reference, table 1 lists the typical imaging parameters and options in an optimized conventional DWI protocol for liver.

In addition to optimizing the parameters for DW-EPI acquisition, the choice of b-values is also important for DWI. However, the optimal b-values used for liver DWI remain unclear, which contributes to the variation of liver ADC reported in the literature. Fundamentally, this is because 
the diffusive motion of water molecules in liver tissue is not conventional free diffusion. Instead, it is mixture of both diffusion and perfusion which will be discussed in more detail later. The change of diffusion-weighted signal cannot be modeled with an exponential function as in equation 1. When the logarithm of diffusion signal change is no longer a linear function of $b$-value, the quantification of ADC becomes b-value dependent. While there is no simple solution due to the limitation of the conventional diffusion model, such problem can be partially addressed by using b-value in the appropriate range. For conventional DWI of liver, at least a low b-value in the range of $0-200 \mathrm{~s} / \mathrm{mm}^{2}$ and a high b-value in the range of $200-1000 \mathrm{~s} / \mathrm{mm}^{2}$ should be included. Kaya et al recommended using 0 and $800 \mathrm{~s} / \mathrm{mm}^{2}$ as two b-values or $0,50,600,800$ and $1000 \mathrm{~s} / \mathrm{mm}^{2}$ as multiple b-values for distinguishing between benign and malignant liver lesions [71]. One factor to keep in mind is that the minimum TE of DW-SS-EPI depends on the largest b-value used. Choosing a larger maximum $b$-value will force a longer TE all the acquisition at all b-values which will reduce the signal due to T2 decay.

One can take advantage the variation with b-value to tailor the DWI for certain type of liver lesions. DWI with low b-value $\left(20 \mathrm{~s} / \mathrm{mm}^{2}\right)$ was reported to demonstrate higher lesion conspicuity of hemangiomas and metastases, than single-shot T2 weighted turbo spin echo (T2W SS TSE) [72]. DWI using a low b-value $\left(50 \mathrm{~s} / \mathrm{mm}^{2}\right)$ was also documented to present a better performance than standard breath-hold T2-weighted imaging for focal liver lesion (FLL) detection [14]. DWI with high b-values (500 and $1000 \mathrm{~s} / \mathrm{mm} 2$ ), on the other hand, could help distinguish hyper-vascular pseudo-lesions from HCCs [73].

While the dependence of b-values may contributes the observed overlap of ADC values between benign and malignant lesions, the similarity of ADC between different pathology could be a more fundamental source of limitation for DWI [43], For example, ADC values of solid benign lesions such as FNH and adenomas may appear similar to malignant lesions such as metastases and HCC, thus limiting the performance of DWI for distinguishing solid liver lesions [22]. 


\section{Advanced Diffusion-weighted Imaging and Applications in Liver}

Despite extensive research in conventional DWI which leads to significant improvement in image quality over time, the ADC values derived from conventional DWI have not been found to correlate well with the characterization of focal liver masses or the prediction of the degree of fibrosis and steatosis. Therefore, more advanced DWI techniques have been developed which hopefully can overcome such limitation.

With the advance in MRI scanner hardware and DWI acquisition strategies, high quality diffusionweighted images with large b-value can be obtained within a short time. The improvements in motion compensation techniques also allow the extension of DWI acquisition over a longer period. Such development makes it possible to perform DWI study with more b-values. By measuring diffusion weighting with multiple b-values over a wider range such as those illustrated in figure 5 and analyzing the data with more sophisticated model, one can not only address the limitation with conventional diffusion model, but also to extract additional information about tissue property and physiology beyond ADC value. Such advanced DWI techniques include Intra-Voxel Incoherent Motion (IVIM), Anomalous Diffusion Imaging (ADI), Diffusion Kurtosis Imaging (DKI) and $\underline{\text { Restriction Spectrum Imaging (RSI). }}$

\section{Intra-Voxel Incoherent Motion (IVIM) Technique}

IVIM is an advanced DWI technique which acquires diffusion-weighted images with multiple bvalues in order to separate and quantify both fast and slow diffusion components [74]. Instead of modeling the attenuation of diffusion-weighted signal with a mono-exponential function, a biexponential function [Equation 2] is used to fit signal intensity dependence on b-value in IVIM as illustrated in figure 6.

$$
\mathbf{S} / \mathbf{S}_{b=0}=(1-\mathrm{PF}) e^{-\mathrm{bD}}+\mathrm{PF} e^{-\mathrm{bD} *}
$$

In IVIM model, the fast component is typically assumed to be originated from the microvascular perfusion while the slow component from free water diffusion. Therefore, fast component is also referred to as pseudo perfusion. Hence, the faction of fast diffusion component is referred to as perfusion fraction $(\mathrm{PF})$. 
IVIM model has been used to characterize liver diffusion in multiple studies. Yoon et al compared IVIM diffusion versus conventional DWI for the staging of liver fibrosis and found IVIM performed better that conventional DWI [34]. Murphy et al demonstrated that liver steatosis and fibrosis have significant independent effects on both IVIM diffusivity (D) and perfusion fraction (PF) [75]. In Murphy's study, only three b-values of 0,100 and $500 \mathrm{~s} / \mathrm{mm}^{2}$ where used and IVIM parameters were calculated essential from the slope of $\ln \left(\mathrm{S}_{\mathrm{S}} \mathrm{S}_{\mathrm{b}=0}\right)$ different $\mathrm{b}$-values instead of biexponential fit. Such analysis would inevitably make IVIM result b-value dependent [76, 77]. Figure 7 shows IVIM results of three clinical cases with different stages of liver fibrosis. The difference in IVIM parameters is consistent with the grade of fibrosis.

Although IVIM has produced promising results in liver application, the reproducibility of measured IVIM diffusivity (D) and perfusion fraction (PF) is relatively poor [78]. This is because fitting the bi-exponential function in equation 2 is highly susceptible to noise and error in the diffusion weighted images and therefore less robust than fitting the mono-exponential function in equation 1. Combination of long acquisition time and poor reproducibility hampers the widespread clinical use of IVIM.

\section{$\underline{\text { Anomalous Diffusion Imaging (ADI) Technique }}$}

In IVIM model, the diffusive motion is separated into two major components. The slow component is often associated with the conventional diffusion while the fast component is associated with a transport process in microscopic level such as blood perfusion in the capillaries. Each component is still assumed to be conventional diffusion, but with its unique diffusion coefficient. Such model, although useful, could be over-simplified. Since water diffusion in tissue is affected by cellular and macromolecular structures forming compartments of different scales and barriers of varying permeability, there could very well be a continuous spectrum of components with varying diffusivity [79]. At the same time, the active transport process from vascular to cellular level is also very different and cannot be described with pseudo diffusion of a single diffusion constant. The movement of water molecules in such complex environment is no longer the classic Brownian motion or normal diffusion where the mean square displacement (MSD), or the amount of space that a water molecule can explore, increases linearly with time. Sub-diffusion occurs when the diffusion process is altered by obstacles that hinder water molecules from moving away. On the other hand, super-diffusion happens when the diffusion process is altered by active transportation 
which carries water molecules along. The non-linear relationships of MSD versus time for superdiffusion and sub-diffusion are illustrated in the figure 8.

Stretched exponential model [Equation 3] was proposed to describe anomalous diffusion in such complex and inhomogeneous environment $[80,81]$.

$$
\mathrm{S} / S_{b=0}=e^{-(b * \mathrm{DDC})^{\alpha}}
$$

Where DDC is the distributed diffusion coefficient and $\alpha$, which is limited to 0 and 1 , can be considered as non-mono-exponential index. When $\alpha=1$, the stretched exponential model is the same as conventional mono-exponential model and, as $\alpha$ approaches 0 , the degree of multiple exponential increases as shown in figure 9. Stretched exponential model currently has only been used to analyze the liver diffusion data acquired from ex-vivo liver specimens [82].

\section{$\underline{\text { Diffusion Kurtosis Imaging (DKI) Technique }}$}

The conventional diffusion model is based on the assumption that the spatial distribution profile of water molecules from diffusion is a Gaussian function. In case of IVIM, there are two Gaussian profiles with narrow and wider width, corresponding to the slow and fast diffusion components with low and high ADCs.

In the complex environment of biological tissue, the influence of tissue structure on water diffusion cannot be fully described as a simple change in ADC. It also causes the distribution profile to deviate from the Gaussian shape. Such non-Gaussianity is called Kurtosis which can be quantified as apparent kurtosis coefficient, $K_{a p p}$ [83-85]. At the same time, a kurtosis corrected diffusion coefficient, $\mathrm{D}_{\mathrm{app}}$, can be derived from the Kurtosis model below.

$$
\mathrm{S} / S_{b=0}=e^{-b * D_{a p p}+\frac{1}{6} b^{2} * D_{a p p}{ }^{2} * K_{a p p}}
$$

If $\mathrm{K}_{\mathrm{app}}$ approaches zero, the Kurtosis model becomes the same as conventional diffusion model and $\mathrm{D}_{\mathrm{app}}=\mathrm{ADC}$.

One of the major challenges for DKI is the need to acquire diffusion weighed images with much higher b-values in the range of $2000-4000 \mathrm{~s} / \mathrm{mm}^{2}$. This is because, only at such high b-values, the non-Gaussian Kurtosis model starts to deviate away from the Gaussian model, as shown in 
figure 10. The loss of signal from both diffusion weighting and long TE could makes such measurement very time consuming as more signal averaging would be required to produce reliable results.

So far, DKI study of liver is limited due to technical challenges discussed previously. Rosenkrantz et al perfomed DKI of ex-vivo liver specimens and found that intermediate-to-substantial excess diffusional kurtosis in HCC as well as significant higher corrected diffusion coefficient $\left(\mathrm{D}_{\mathrm{app}}\right)$ than conventional ADC [86]. More recently, Anderson et al studied the possibility of evaluating liver fibrosis with DKI with ex-vivo murine liver specimens at $9.4 \mathrm{~T}$ and found significant correlation between kurtosis model parameters $\left(\mathrm{D}_{\mathrm{app}}, \mathrm{K}_{\mathrm{app}}\right)$ and liver fibrosis [82]. Although DKI has been applied in human brain and even prostate studies, there is only one published liver DKI study of human subjects to our knowledge [87]. .In this study, the authors measured mean kurtosis (MK) of hypervascular hypercelluar carcinoma (HCC) successfully with six b-values of $0-2000 \mathrm{~s} / \mathrm{mm}^{2}$ and found that mean kurtosis (MK) performed better than ADC in differentiating viable versus non-viable HCC. Unfortunately, no IVIM analysis was done and compared with DKI to determine if DKI has any advantage over IVIM.

\section{$\underline{\text { Restriction Spectrum Imaging (RSI) Technique }}$}

RSI is a general frame work for modeling advanced diffusion weighted imaging [88]. It assumes the acquired signal is a linear combination of a distribution of pools or components of water in the tissue with different diffusion characteristics, such as free diffusion, hindered diffusion and restricted diffusion. The goal is to determine the relative fraction of each component and as well as its diffusion parameters, which are influenced by tissue properties such as cell size, density, orientation, etc. In order to accomplish such goal, a wide range of diffusion weighted images with varying b-values and diffusion encoding orientations need to be acquired. Such requirement leads to the similar challenges in liver imaging application as other advanced DWI techniques discussed previously, i.e. its very time consuming. Therefore, RSI is so far only limited to brain and prostate applications.

\section{Number and distribution of b-values}

For advanced DWI techniques that acquires data of multiple b-values, choice of the number of bvalues as well as their range and distribution becomes an important issues. While more data points 
with different b-values allow better sampling of the diffusion signal model, it also increases the acquisition time. Such trade-off is application dependent and should be carefully considered. The number of b-values ranging from 7 to 20 is typically used for abdominal imaging studies. Although the fewer b-values tend to increase the error of the estimated IVIM parameters, it was reported that liver IVIM with as few as 4 b-values did not substantially compromise the precision and reproducibility of IVIM parameters compared to the acquisition of $16 \mathrm{~b}$-values [89]. Given the constraint from the number of b-values, the range and distribution should also be carefully considered to make the modeling more robust. Take the two-step approach for example, several high b-values should be included to reliably determine the $\mathrm{D}$ or $\mathrm{D}_{\text {slow }}$ parameter and several low b-values should be include to be sensitive to the influence of $\mathrm{D}^{*}$ or $\mathrm{D}_{\text {fast }}$ component.

\section{Multi-shot High-resolution Liver DWI Technique}

In order to avoid motion-related artifacts, liver DWI is typically acquired with SS-EPI as discussed previously. SS-EPI based DWI protocols, however, does have some limitations, including limited spatial resolution; and SNR penalty due to long EPI echo train. Such limitations inevitably compromise the image quality of liver DWI and make it more challenging to detect subtle lesions with DW-SS-EPI. Recently, there have been some attempts to high resolution liver DWI with multi-shot or segmented EPI.

Tokoro et al used the so called readout-segment EPI (RESOLVE) which divides the k-space into multiple segments with the same number of phase encoding but fewer data points in readout direction [90]. A 2D navigator echo is re-acquired with each segment to detect and correct phase changes between multiple segments. They found FB RESOVLE causes fewer artifacts and allows comparable lesion detectability versus RT DW-SS-EPI with shorter scan time [91].

More recently, Chang et al applied another multi-shot technique called multiplexed sensitivity encoded (MUSE) and combined it with either navigator-based respiratory-gating or breath hold for liver DWI [92]. The more anatomical details could be visualized in multi-shot MUSE DWI [93].

\section{Advanced DWI Post-processing and Analysis}


There are several different approaches for fitting the bi-exponential function to IVIM. One-step approach uses all the data points with different b-values in order to find the best fit. However, due to the larger degree of freedom, the search may ends up in a local rather than a global minimum of the parameter space, especially of the initial condition has a large deviation. Such error is more likely to occur when the quality of data is suboptimal, i.e. compromised by noise or artifacts. Therefore, a two-step approach is now commonly used.

With two-step approach, the data points with relatively higher b-value $\left(b=>200 \mathrm{~s} / \mathrm{mm}^{2}\right)$ is fitted with a mono-exponential function first to determine the $\mathrm{D}$ or $\mathrm{D}_{\text {slow. }}$ Then, the $\mathrm{D}$ or $\mathrm{D}_{\text {slow }}$ parameter is fixed or locked when fitting all the data points with the bi-exponential function. While two-step approach is shown to improve the stability of fitting, it will also unavoidably introduce biases in the results.

\section{Texture and Histogram Analysis}

Another idea of extracting information related to tissue diffusion properties for diagnosis and evaluation is through texture or histogram analysis. With texture analysis, a set of metrics characterizing the perceived texture of an image is calculated. With histogram analysis, the heterogeneity or the distribution of image intensity within a region of interest is evaluated. While both texture and histogram analysis can be applied to the results of liver DWI such as the ADC map, the discussion of the methods of texture and histogram analysis is beyond the scope of this review. Barry et al applied texture analysis to the ex-vivo murine liver ADC map and found moderate to very strong correlation between texture features and liver fibrosis [94]. Lambregts et al performed histogram analysis of ADC values of the whole liver parenchyma, excluding focal lesions, in patients with proven colorectal liver metastases and found a significant difference in fifth percentile and standard deviation compared with healthy controls without liver disease [95]. Such results suggest that the changes in liver microarchitecture and diffusion properties may occur not only at the site of focal metastatic lesions visible on imaging but also in the liver parenchyma that appears to be free from tumor on MRI.

\section{Discussion and Conclusions}

Conventional DWI provides useful diagnostic information in liver imaging. The images can be acquired within a short amount time for two to three b-values even with respiratory triggering. The 
artifact can be minimized with latest fat suppression techniques and optimized EPI acquisition parameters even at $3 \mathrm{~T}$. The calculation of ADC is straight forward and is ready available as part of the software on the scanner console. Therefore, conventional DWI has been widely integrated in routine clinical imaging of the liver.

Despite the success of conventional diffusion-weighted imaging and its clinical application, diffusion-weighted imaging is continuing to advance rapidly. The improvement of scanner performance and acquisition techniques provides high quality DWI images and allows more sophisticated models to be used to analyze DWI data. Such model can potentially better characterize water diffusion in the complicated in-vivo environment and the addition diffusion parameters beyond the conventional ADC maybe more sensitive to physiological changes in the disease process and therefore of value for clinical diagnosis. However, there are two major technical challenges with the advanced DWI of the liver. One of them is due to the requirement of more images with incrementing $b$-values and also images with high $b$-values $\left(b>1000 \mathrm{~s} / \mathrm{mm}^{2}\right)$ where more signal averaging is needed to compensate for low SNR. Such requirement prolongs the acquisition time of advanced DWI of liver. Because of the long scan time, clinical efficiency and patient cooperation becomes the obstacle for its routine use. Although higher field strength such as 3.0T offers greater SNR, its advantage in liver DWI over $1.5 \mathrm{~T}$ is limited. This is because EPI acquisition is sensitive to the local magnetic field inhomogeneity which also increases with field strength. In order to reduce the artifacts resulting from local magnetic field inhomogeneity, certain adjustments in the imaging parameters need to be made which also compromises the SNR gain from 3.0T. Therefore, new technical advances to accelerate the DWI acquisition such as simultaneous multiple slice imaging is holds greater promise in making advanced DWI of liver clinically feasible. Another challenge is the choice of an appropriate model and the understanding of the physiological basis or justification for such model. Except for IVIM, the appropriateness of other advanced DWI models for liver application remains to be investigated. At this stage, the modeling of advanced DWI is typically done offline with software tools developed in individual research labs. Overall, there has been limited experience of these advanced DWI technique and its application in liver that the optimized protocols and standard post-processing are not established. Further studies are needed to carefully compare different techniques and to evaluate their benefits and value in clinical diagnosis. 


\section{References:}

1. Padhani, A.R., et al., Diffusion-weighted magnetic resonance imaging as a cancer biomarker: consensus and recommendations. Neoplasia, 2009. 11(2): p. 102-25.

2. An, C., S. Park, and Y.J. Choi, Diffusion-weighted MRI in intrahepatic bile duct adenoma arising from the cirrhotic liver. Korean J Radiol, 2013. 14(5): p. 769-75.

3. Palmucci, S., et al., Diffusion-weighted MRI in a liver protocol: its role in focal lesion detection. World J Radiol, 2012. 4(7): p. 302-10.

4. Kim, Y.K., et al., Noncontrast MRI with diffusion-weighted imaging as the sole imaging modality for detecting liver malignancy in patients with high risk for hepatocellular carcinoma. Magn Reson Imaging, 2014. 32(6): p. 610-8.

5. Kang, T.W., et al., Inflammatory myofibroblastic tumours of the liver: gadoxetic acidenhanced and diffusion-weighted MRI findings with 18F-FDG PET/CT and clinical significance of regression on follow-up. Clin Radiol, 2014. 69(5): p. 509-18.

6. Lee, M.H., et al., Gadoxetic acid-enhanced hepatobiliary phase MRI and high-b-value diffusion-weighted imaging to distinguish well-differentiated hepatocellular carcinomas from benign nodules in patients with chronic liver disease. AJR Am J Roentgenol, 2011. 197(5): p. W868-75.

7. Hardie, A.D., M.K. Kizziah, and D.J. Boulter, Diagnostic accuracy of diffusion-weighted MRI for identifying hepatocellular carcinoma with liver explant correlation. J Med Imaging Radiat Oncol, 2011. 55(4): p. 362-7.

8. Chung, J., et al., Hypervascular hepatocellular carcinoma in the cirrhotic liver: diffusionweighted imaging versus superparamagnetic iron oxide-enhanced MRI. Magn Reson Imaging, 2011. 29(9): p. 1235-43.

9. Sinitsyn, V.E. and N.A. Chueva, [Differential diagnosis of liver focal formations at diffusion MRI]. Eksp Klin Gastroenterol, 2010(7): p. 131-9.

10. Donati, F., et al., Focal nodular hyperplasia of the liver: diffusion and perfusion MRI characteristics. Magn Reson Imaging, 2013. 31(1): p. 10-6.

11. Haradome, H., et al., T2-weighted and diffusion-weighted MRI for discriminating benign from malignant focal liver lesions: diagnostic abilities of single versus combined interpretations. J Magn Reson Imaging, 2012. 35(6): p. 1388-96.

12. Fruehwald-Pallamar, J., et al., Confident non-invasive diagnosis of pseudolesions of the liver using diffusion-weighted imaging at 3T MRI. Eur J Radiol, 2012. 81(6): p. 1353-9.

13. Oruc, E., et al., The role of diffusion-weighted MRI in the classification of liver hydatid cysts and differentiation of simple cysts and abscesses from hydatid cysts. Diagn Interv Radiol, 2010. 16(4): p. 279-87.

14. Parikh, T., et al., Focal liver lesion detection and characterization with diffusion-weighted MR imaging: comparison with standard breath-hold T2-weighted imaging. Radiology, 2008. 246(3): p. 812-822.

15. Tappouni, R., et al., Comparison of the accuracy of diffusion-weighted imaging versus dynamic contrast enhancement magnetic resonance imaging in characterizing focal liver lesions. $\mathrm{J}$ Comput Assist Tomogr, 2013. 37(6): p. 995-1001.

16. Kim, Y.K., et al., Noncontrast MRI with diffusion-weighted imaging as the sole imaging modality for detecting liver malignancy in patients with high risk for hepatocellular carcinoma. Magn Reson Imaging, 2014. 32(6): p. 610-618. 
17. Chandarana, H. and B. Taouli, Diffusion-weighted MRI and liver metastases. Magn Reson Imaging Clin N Am, 2010. 18(3): p. 451-64, x.

18. Hardie, A.D., et al., Diagnosis of liver metastases: value of diffusion-weighted MRI compared with gadolinium-enhanced MRI. Eur Radiol, 2010. 20(6): p. 1431-41.

19. Shimada, K., et al., Comparison of gadolinium-EOB-DTPA-enhanced and diffusion-weighted liver MRI for detection of small hepatic metastases. Eur Radiol, 2010. 20(11): p. 2690-8.

20. Yu, M.H., et al., Gadoxetic acid-enhanced MRI and diffusion-weighted imaging for the detection of colorectal liver metastases after neoadjuvant chemotherapy. Eur Radiol, 2015.

21. Koh, D.M., et al., Combining diffusion-weighted MRI with Gd-EOB-DTPA-enhanced MRI improves the detection of colorectal liver metastases. Br J Radiol, 2012. 85(1015): p. 980-9.

22. Miller, F.H., et al., Utility of diffusion-weighted MRI in distinguishing benign and malignant hepatic lesions. J Magn Reson Imaging, 2010. 32(1): p. 138-147.

23. Sutherland, T., et al., Solid focal liver lesion characterisation with apparent diffusion coefficient ratios. J Med Imaging Radiat Oncol, 2014. 58(1): p. 32-37.

24. Parsai, A., et al., Assessment of diffusion-weighted imaging for characterizing focal liver lesions. Clin Imaging, 2015. 39(2): p. 278-284.

25. Donati, O.F., et al., Accuracy and confidence of Gd-EOB-DTPA enhanced MRI and diffusionweighted imaging alone and in combination for the diagnosis of liver metastases. Eur J Radiol, 2013. 82(5): p. 822-8.

26. Lee, M.H., et al., Gadoxetic acid-enhanced hepatobiliary phase MRI and high-b-value diffusion-weighted imaging to distinguish well-differentiated hepatocellular carcinomas from benign nodules in patients with chronic liver disease. AJR Am J Roentgenol, 2011. 197(5): p. W868-875.

27. Schmid-Tannwald, C., et al., Diffusion-weighted MRI Before and After Robotic Radiosurgery (Cyberknife(R)) in Primary and Secondary Liver Malignancies: A Pilot Study. Technol Cancer Res Treat, 2015. 14(2): p. 191-9.

28. Padhani, A.R. and D.M. Koh, Diffusion MR imaging for monitoring of treatment response. Magn Reson Imaging Clin N Am, 2011. 19(1): p. 181-209.

29. Kamel, I.R., et al., Unresectable hepatocellular carcinoma: serial early vascular and cellular changes after transarterial chemoembolization as detected with MR imaging. Radiology, 2009. 250(2): p. 466-473.

30. Lu, T.L., et al., Assessment of liver tumor response by high-field (3 T) MRI after radiofrequency ablation: short- and mid-term evolution of diffusion parameters within the ablation zone. Eur J Radiol, 2012. 81(9): p. e944-950.

31. Malayeri, A.A., et al., Principles and applications of diffusion-weighted imaging in cancer detection, staging, and treatment follow-up. Radiographics, 2011. 31(6): p. 1773-1791.

32. Palmucci, S., et al., Diffusion-Weighted MRI for the Assessment of Liver Fibrosis: Principles and Applications. Biomed Res Int, 2015. 2015: p. 874201.

33. Taouli, B., et al., Diffusion-weighted MRI for quantification of liver fibrosis: preliminary experience. AJR Am J Roentgenol, 2007. 189(4): p. 799-806.

34. Yoon, J.H., et al., Evaluation of hepatic fibrosis using intravoxel incoherent motion in diffusion-weighted liver MRI. J Comput Assist Tomogr, 2014. 38(1): p. 110-6.

35. Kovac, J.D., et al., Diffusion-weighted MRI versus transient elastography in quantification of liver fibrosis in patients with chronic cholestatic liver diseases. Eur J Radiol, 2012. 81(10): p. 2500-6. 
36. Soylu, A., et al., Utility of diffusion-weighted MRI for assessing liver fibrosis in patients with chronic active hepatitis. Diagn Interv Radiol, 2010. 16(3): p. 204-8.

37. Sandrasegaran, K., et al., Value of diffusion-weighted MRI for assessing liver fibrosis and cirrhosis. AJR Am J Roentgenol, 2009. 193(6): p. 1556-60.

38. Cece, H., et al., The use of DWI to assess spleen and liver quantitative ADC changes in the detection of liver fibrosis stages in chronic viral hepatitis. Eur J Radiol, 2013. 82(8): p. e30712.

39. Bülow, R., et al., Diffusion-weighted magnetic resonance imaging for staging liver fibrosis is less reliable in the presence of fat and iron. Eur Radiol, 2013. 23(5): p. 1281-1287.

40. Leitao, H.S., et al., Fat deposition decreases diffusion parameters at MRI: a study in phantoms and patients with liver steatosis. Eur Radiol, 2013. 23(2): p. 461-7.

41. Poyraz, A.K., et al., Diffusion-weighted MRI of fatty liver. J Magn Reson Imaging, 2012. 35(5): p. 1108-11.

42. Sandrasegaran, K., et al., Diffusion-weighted MRI of the transplanted liver. Clin Radiol, 2011. 66(9): p. 820-5.

43. Taouli, B. and D.M. Koh, Diffusion-weighted MR imaging of the liver. Radiology, 2010. 254(1): p. 47-66.

44.Kele, P.G. and E.J. van der Jagt, Diffusion weighted imaging in the liver. World J Gastroenterol, 2010. 16(13): p. 1567-76.

45. Le Bihan, D., et al., Imaging of diffusion and microcirculation with gradient sensitization: design, strategy, and significance. J Magn Reson Imaging, 1991. 1(1): p. 7-28.

46. Taouli, B., et al., Parallel imaging and diffusion tensor imaging for diffusion-weighted MRI of the liver: preliminary experience in healthy volunteers. AJR Am J Roentgenol, 2004. 183(3): p. 677-80.

47.Oner, A.Y., et al., Single breath-hold diffusion-weighted MRI of the liver with parallel imaging: initial experience. Clin Radiol, 2006. 61(11): p. 959-65.

48. Filli, L., et al., Simultaneous Multislice Echo Planar Imaging With Blipped Controlled Aliasing in Parallel Imaging Results in Higher Acceleration: A Promising Technique for Accelerated Diffusion Tensor Imaging of Skeletal Muscle. Invest Radiol, 2015.

49. Himanshu Bhat, J.C., Daniel Cornfeld, Ralph Strecker, Bruce Spottiswoode, Frank H Miller, Charles Fasanati, Stephen Cauley, Kawin Setsompop, Keith Heberlein, Accelerated diffusion weighted imaging in the liver with blipped CAIPIRINHA based simultaneous multi slice acquisition. Proc. Intl. Soc. Mag. Reson. Med., 2013. 21(0593).

50. Chika C Obele, C.G., Justin Ream, Ankur Doshi, Naomi Campbell, Cheung Hoi Zhang, James Babb, Himanshu Bhat, Hersh Chandarana, Simultaneous Multislice Accelerated FreeBreathing Diffusion-Weighted Imaging of the Liver at 3T. Proc. Intl. Soc. Mag. Reson. Med., 2015. 23(4016).

51. Ozaki, M., et al., Motion artifact reduction of diffusion-weighted MRI of the liver: use of velocity-compensated diffusion gradients combined with tetrahedral gradients. J Magn Reson Imaging, 2013. 37(1): p. 172-8.

52. Larsen, N.E., et al., Quantitative liver ADC measurements using diffusion-weighted MRI at 3 Tesla: evaluation of reproducibility and perfusion dependence using different techniques for respiratory compensation. MAGMA, 2013. 26(5): p. 431-42.

53. Barnwell, J.D., J.K. Smith, and M. Castillo, Utility of navigator-prospective acquisition correction technique (PACE) for reducing motion in brain MR imaging studies. AJNR Am J Neuroradiol, 2007. 28(4): p. 790-1. 
54. Taouli, B., et al., Diffusion-weighted imaging of the liver: comparison of navigator triggered and breathhold acquisitions. J Magn Reson Imaging, 2009. 30(3): p. 561-8.

55. Kandpal, H., et al., Respiratory-triggered versus breath-hold diffusion-weighted MRI of liver lesions: comparison of image quality and apparent diffusion coefficient values. AJR Am J Roentgenol, 2009. 192(4): p. 915-22.

56. Nasu, K., et al., Hepatic pseudo-anisotropy: a specific artifact in hepatic diffusion-weighted images obtained with respiratory triggering. MAGMA, 2007. 20(4): p. 205-11.

57. Neil Peter Jerome, E.K., Matthew R Orton, James A d'Arcy, Bernd Kuehn, Dow-Mu Koh, David J Collins, Martin O Leach, Abdominal diffusion imaging parameters from freebreathing multiple-averaged and finely-sampled decay curves compared to acquisition using active breathing control. Proc. Intl. Soc. Mag. Reson. Med., 2015. 23(1615).

58. Kwee, T.C., et al., Diffusion-weighted whole-body imaging with background body signal suppression (DWIBS): features and potential applications in oncology. Eur Radiol, 2008. 18(9): p. 1937-52.

59. Alison Sandberg, T.P., Glyn Johnson, Alto Stemmer, Jian Xu, Bachir Taouli, Feasibility of a Respiratory-Triggered SSEPI Diffusion-Weighted Sequence for Liver Imaging Using Navigator Echo Technique: Comparison with Breath-Hold Diffusion-Weighted Sequence. Proc. Intl. Soc. Mag. Reson. Med., 2006. 14(400).

60. Kwee, T.C., et al., Comparison and reproducibility of ADC measurements in breathhold, respiratory triggered, and free-breathing diffusion-weighted MR imaging of the liver. J Magn Reson Imaging, 2008. 28(5): p. 1141-8.

61. Oi Lei Wong, G.G.L., Jing Yuan, Wai Kit Chung, Max W. K. Law, Benny W. H. Ho, Michael D. Noseworthy, Evaluation of Pseudo-Hepatic Anisotropy Artifact in Liver Intravoxel Incoherent Motion (IVIM) based on Clustering Technique. Proc. Intl. Soc. Mag. Reson. Med., 2015. 23(3998).

62. Nasu, K., et al., The effect of simultaneous use of respiratory triggering in diffusion-weighted imaging of the liver. Magn Reson Med Sci, 2006. 5(3): p. 129-36.

63. Naganawa, S., et al., Diffusion-weighted imaging of the liver: technical challenges and prospects for the future. Magn Reson Med Sci, 2005. 4(4): p. 175-86.

64. Bruegel, M., et al., Characterization of focal liver lesions by ADC measurements using a respiratory triggered diffusion-weighted single-shot echo-planar MR imaging technique. Eur Radiol, 2008. 18(3): p. 477-85.

65. Murtz, P., et al., Abdomen: diffusion-weighted MR imaging with pulse-triggered single-shot sequences. Radiology, 2002. 224(1): p. 258-64.

66. Liau, J., et al., Cardiac motion in diffusion-weighted MRI of the liver: artifact and a method of correction. J Magn Reson Imaging, 2012. 35(2): p. 318-27.

67. Hernando, D., et al., Removal of olefinic fat chemical shift artifact in diffusion MRI. Magn Reson Med, 2011. 65(3): p. 692-701.

68. Burakiewicz, J., et al., Water-fat separation in diffusion-weighted EPI using an IDEAL approach with image navigator. Magn Reson Med, 2015. 73(3): p. 964-72.

69. Lee, J.S., et al., Diffusion-weighted imaging of the liver at $3 \mathrm{~T}$ using section-selection gradient reversal: emphasis on chemical shift artefacts and lesion conspicuity. Clin Radiol, 2015. 70(4): p. $408-15$.

70. Haibo Zhang, H.X., Hui Liu, Stemmer Alto, Kannengiesser Stephan, Kiefer Berthold, and Zhengyu Jin, Improved lesion detection in regions with strong susceptibility using iShim- 
WBDWI as compared to 3D-Shimming WBDWI. Proc. Intl. Soc. Mag. Reson. Med., 2015. 23(3993).

71. Kaya, B. and Z. Koc, Diffusion-weighted MRI and optimal b-value for characterization of liver lesions. Acta Radiol, 2014. 55(5): p. 532-42.

72. Coenegrachts, K., et al., Improved focal liver lesion detection: comparison of single-shot diffusion-weighted echoplanar and single-shot T2 weighted turbo spin echo techniques. Br J Radiol 2007. 80(955): p. 524-531.

73. Motosugi, U., et al., Distinguishing hypervascular pseudolesions of the liver from hypervascular hepatocellular carcinomas with gadoxetic acid-enhanced MR imaging. Radiology 2010. 256(1): p. 151-158.

74. Le Bihan, D., et al., Separation of diffusion and perfusion in intravoxel incoherent motion MR imaging. Radiology, 1988. 168(2): p. 497-505.

75. Murphy, P., et al., Associations between histologic features of nonalcoholic fatty liver disease (NAFLD) and quantitative diffusion-weighted MRI measurements in adults. J Magn Reson Imaging, 2014.

76. Taimouri, V., et al., Spatially constrained incoherent motion method improves diffusionweighted MRI signal decay analysis in the liver and spleen. Med Phys, 2015. 42(4): p. 1895.

77. Penner, A.H., et al., Intravoxel incoherent motion model-based liver lesion characterisation from three b-value diffusion-weighted MRI. Eur Radiol, 2013. 23(10): p. 2773-83.

78. Kakite, S., et al., Hepatocellular carcinoma: short-term reproducibility of apparent diffusion coefficient and intravoxel incoherent motion parameters at 3.0T. J Magn Reson Imaging, 2015. 41(1): p. 149-56.

79. Hall, M.G. and T.R. Barrick, From diffusion-weighted MRI to anomalous diffusion imaging. Magn Reson Med, 2008. 59(3): p. 447-55.

80. Bennett, K.M., et al., Characterization of continuously distributed cortical water diffusion rates with a stretched-exponential model. Magn Reson Med, 2003. 50(4): p. 727-34.

81. Hoff, B.A., et al., Assessment of multiexponential diffusion features as MRI cancer therapy response metrics. Magn Reson Med, 2010. 64(5): p. 1499-509.

82. Anderson, S.W., et al., Characterizing non-gaussian, high b-value diffusion in liver fibrosis: Stretched exponential and diffusional kurtosis modeling. J Magn Reson Imaging, 2014. 39(4): p. 827-34.

83. Jensen, J.H., et al., Diffusional kurtosis imaging: the quantification of non-gaussian water diffusion by means of magnetic resonance imaging. Magn Reson Med, 2005. 53(6): p. 143240.

84. Jensen, J.H. and J.A. Helpern, MRI quantification of non-Gaussian water diffusion by kurtosis analysis. NMR Biomed, 2010. 23(7): p. 698-710.

85. Rosenkrantz, A.B., et al., Body diffusion kurtosis imaging: Basic principles, applications, and considerations for clinical practice. J Magn Reson Imaging, 2015.

86. Rosenkrantz, A.B., et al., Assessment of hepatocellular carcinoma using apparent diffusion coefficient and diffusion kurtosis indices: preliminary experience in fresh liver explants. Magn Reson Imaging, 2012. 30(10): p. 1534-40.

87. Goshima, S., et al., Diffusion kurtosis imaging to assess response to treatment in hypervascular hepatocellular carcinoma. AJR Am J Roentgenol, 2015. 204(5): p. W543-9.

88. White, N.S., et al., Diffusion-weighted imaging in cancer: physical foundations and applications of restriction spectrum imaging. Cancer Res, 2014. 74(17): p. 4638-52. 
89. Dyvorne, H., et al., Intravoxel incoherent motion diffusion imaging of the liver: optimal bvalue subsampling and impact on parameter precision and reproducibility. Eur J Radiol, 2014. 83(12): p. 2109-13.

90. Porter, D.A. and R.M. Heidemann, High resolution diffusion-weighted imaging using readoutsegmented echo-planar imaging, parallel imaging and a two-dimensional navigator-based reacquisition. Magn Reson Med, 2009. 62(2): p. 468-75.

91. Tokoro, H., et al., Usefulness of free-breathing readout-segmented echo-planar imaging (RESOLVE) for detection of malignant liver tumors: comparison with single-shot echo-planar imaging (SS-EPI). Eur J Radiol, 2014. 83(10): p. 1728-33.

92. Chen, N.K., et al., A robust multi-shot scan strategy for high-resolution diffusion weighted MRI enabled by multiplexed sensitivity-encoding (MUSE). Neuroimage, 2013. 72: p. 41-7.

93. Hing-Chiu Chang, A.G., Dan Xu, Lloyd Estkowski, Ersin Bayram, Mei-Lan Chu, Mustafa R Bashir, Allen W Song, and Nan-kuei Chen, High-resolution abdominal diffusion-weighted imaging based on multi-shot and multiplexed sensitivity encoded echo-planar imaging. Proc. Intl. Soc. Mag. Reson. Med., 2015. 23(4015).

94. Barry, B., et al., Quantifying liver fibrosis through the application of texture analysis to diffusion weighted imaging. Magn Reson Imaging, 2014. 32(1): p. 84-90.

95. Lambregts, D.M., et al., Whole-liver diffusion-weighted MRI histogram analysis: effect of the presence of colorectal hepatic metastases on the remaining liver parenchyma. Eur $\mathrm{J}$ Gastroenterol Hepatol, 2015. 27(4): p. 399-404. 


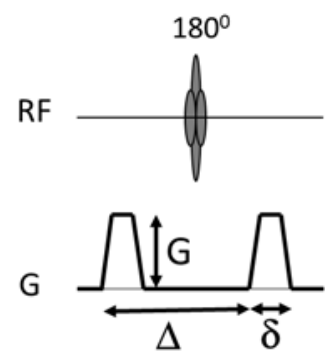

Figure 1: Diffusion sensitizing module with Stejskal-Tanner diffusion scheme where two mono-polar gradient pulses are separated by an $180^{\circ}$ refocusing RF pulse. The degree of diffusion weighting depends on the amplitude $(\mathrm{G})$ and width $(\delta)$ of gradient pulses as well as the separation or delay $(\Delta)$ between the two gradient pluses.

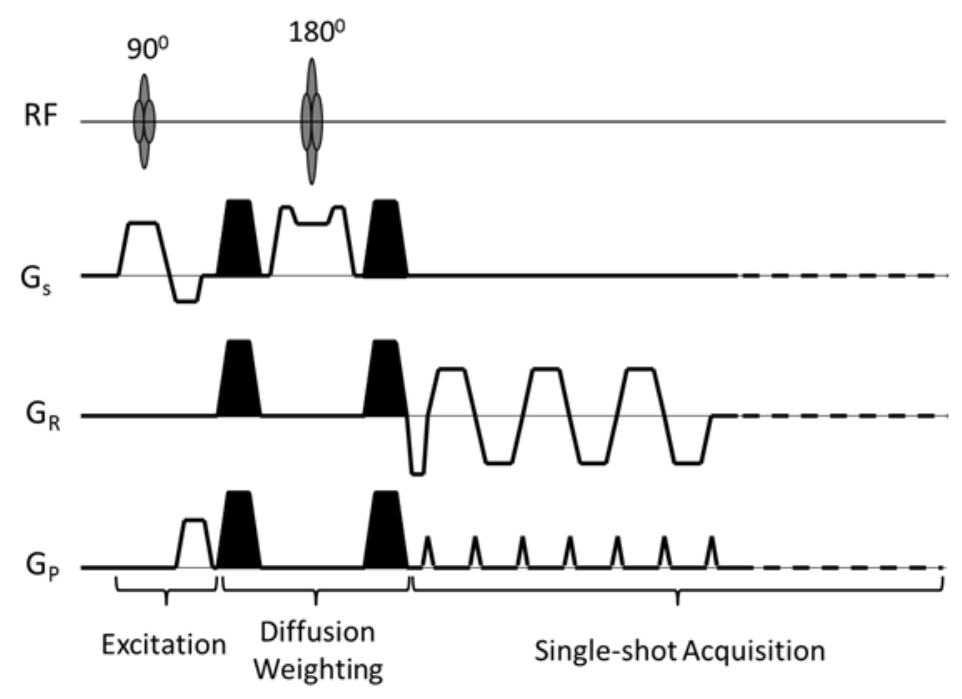

Figure 2: DW-SS-EPI pulse sequence where single-shot EPI acquisition is preceded by a slice selective excitation of $90^{\circ}$ flip angle and a diffusion weighting module discussed previously. 

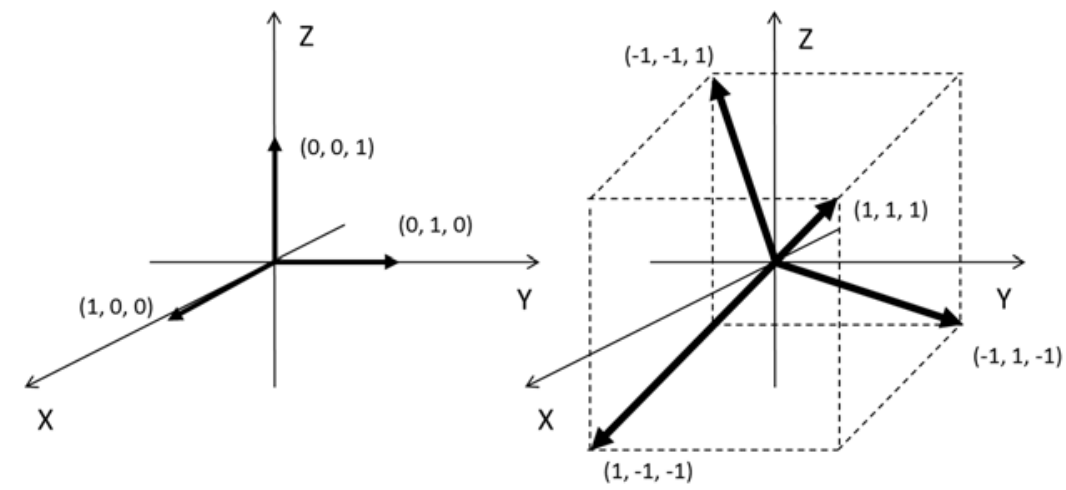

Figure 3. Orthogonal and tetrahedral diffusion weighting. In orthogonal diffusion weighting, the diffusion sensitizing gradients are turned on for one direction at a time to produce diffusion weighting vectors of $(1,0,0),(0,1,0)$ and $(0,0,1)$. In tetrahedral diffusion weighting, the diffusion sensitizing gradients in all three directions are turned on at same time to produce diffusion weighting vectors of $(1,-1,-1),(-1,1,-1),(1,1,1)$ and $(-1,-1,1)$. With tetrahedral diffusion weighting, the amplitude of effective diffusion weighting gradient is $\sqrt{3}(\sim 1.73)$ times greater than orthogonal diffusion weighting under the same gradient performance.

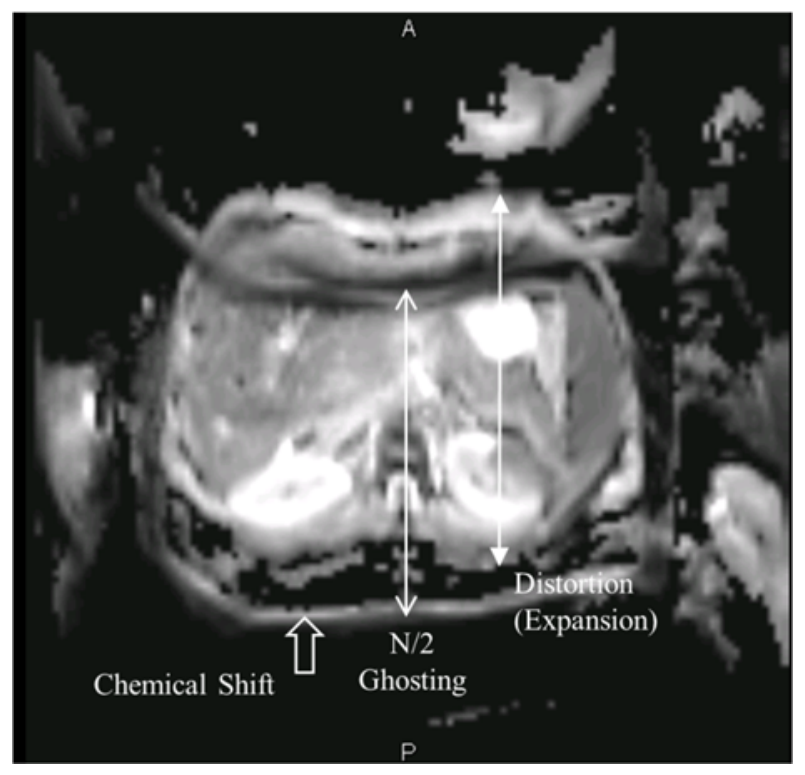

Figure 4: Chemical shift, N/2 or Nyquist ghosting and geometric distortion artifacts are commonly observed with DW-SS-EPI in liver application. The because of chemical shift the signal from fat, if not completely suppressed, can form a band of interference over the liver. Nyquist ghosting can cause a fraction of the liver signal to be shifted half FOV in the phase encoding direction over overlay on the correct image. The geometric distortion can cause signal pile up in certain areas. It also makes correlating DWI images with 
other types of images more challenging. All of these artifacts will compromise quality of diffusion weighed images and introduce error in the quantification.

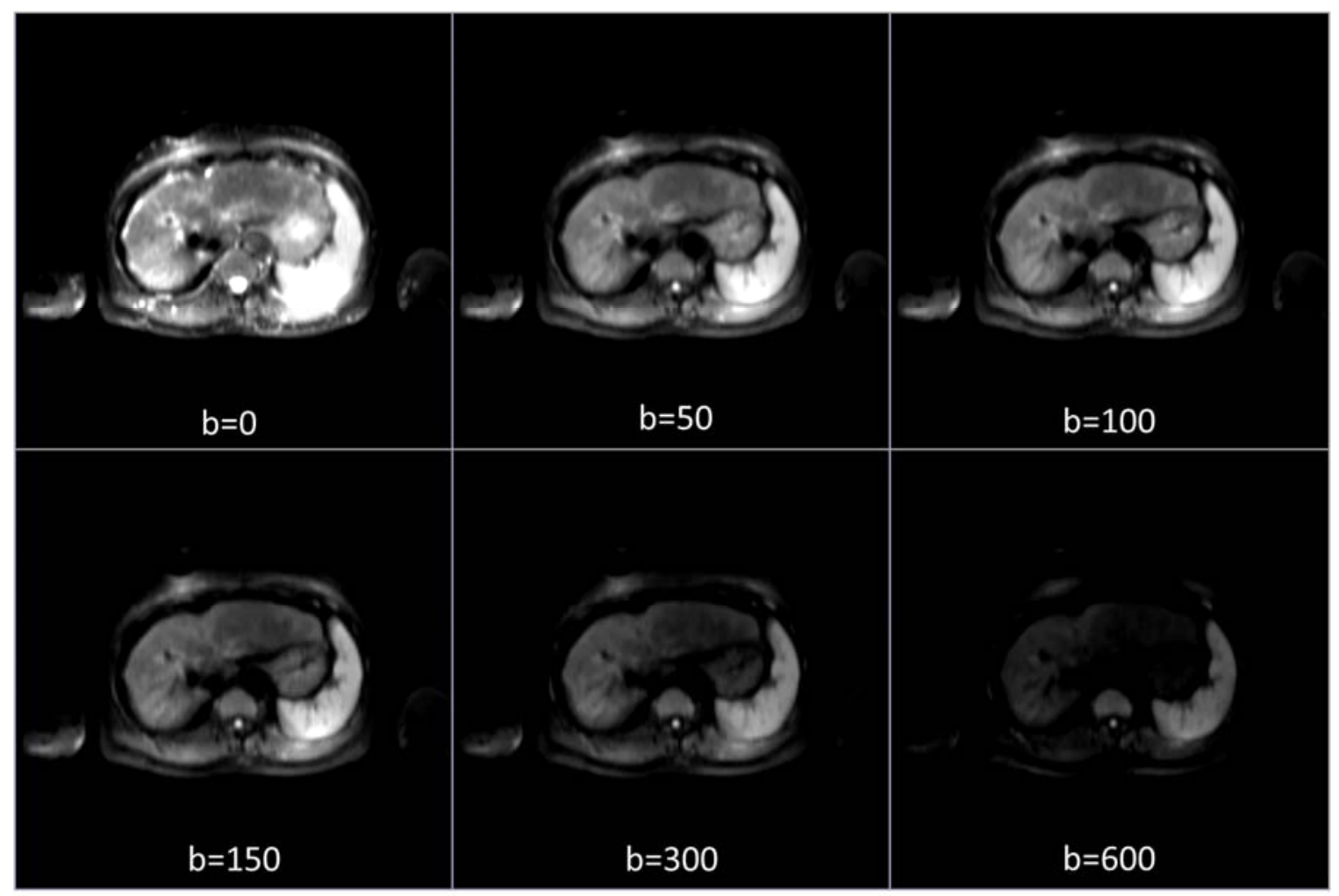

Figure 5. Typical liver IVIM DWI images with six different b-values of $0-600 \mathrm{~s} / \mathrm{mm}^{2}$ acquired with respiratory triggering at $1.5 \mathrm{~T}$.

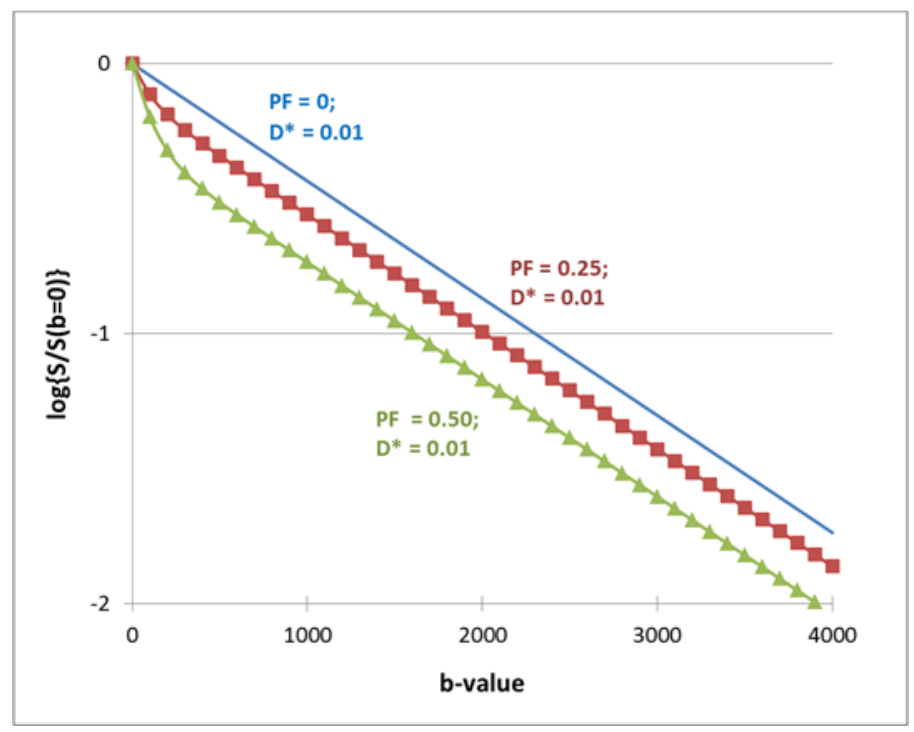


Figure 6. IVIM diffusion model of $\mathrm{PF}=0.25 ; \mathrm{D}^{*}=0.01$ and $\mathrm{PF}=0.5 ; \mathrm{D}^{*}=0.01$ versus conventional diffusion model of $P F=0$. With the increase of perfusion fraction $(\mathrm{PF})$, the attenuation of diffusion weighted signal with respect to b-value in IVIM models deviates from the conventional diffusion model (the straight blue line) mostly in the low b-value range.
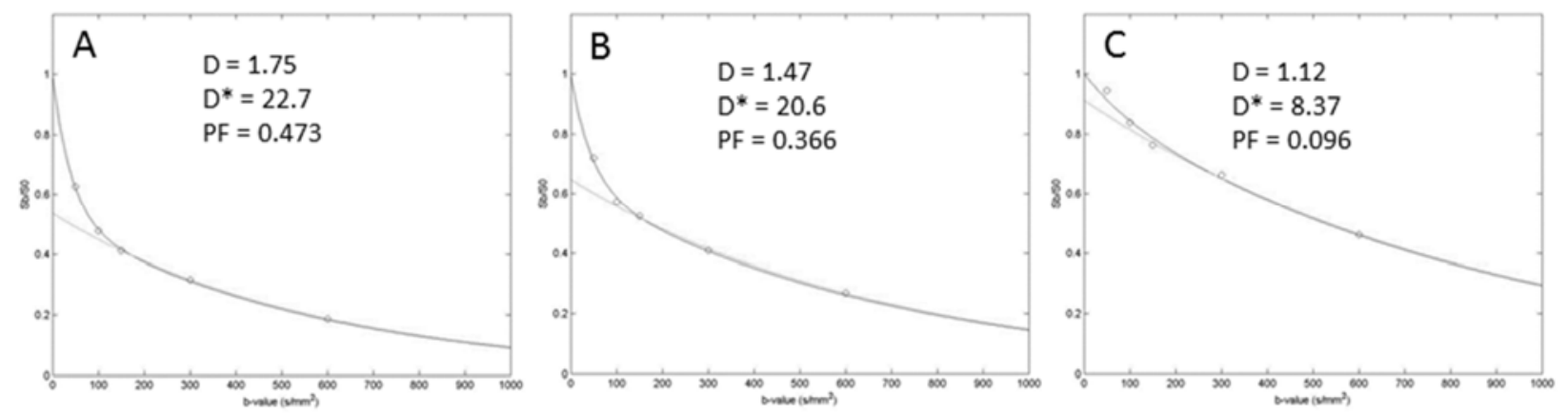

Figure 7. Plots of relative signal intensity at different $b$-values (signal at $b=0 \mathrm{~s} / \mathrm{mm}^{2}$ is 1 ) in $y$-axis versus b-values on x-axis for different patients. A. 61-year-old male with elevated liver function and no biopsy evidence of liver fibrosis (F0). Note the high D, D* and PF values indicating relatively unhampered diffusion and perfusion. B. 51-year-old male with hepatitis B and found to have F1 fibrosis on biopsy. Note the reduction in $\mathrm{D}, \mathrm{D}^{*}$ and $\mathrm{PF}$ compared to patient A. C. 54-year-old male with clinically established cirrhosis and biopsy finding of F4. Note that $\mathrm{D}, \mathrm{D}^{*}$ and PF values are the lowest in this patient. In general IVIM parameters, especially $\mathrm{D}^{*}$, have been shown to correlate well with grades of liver fibrosis.

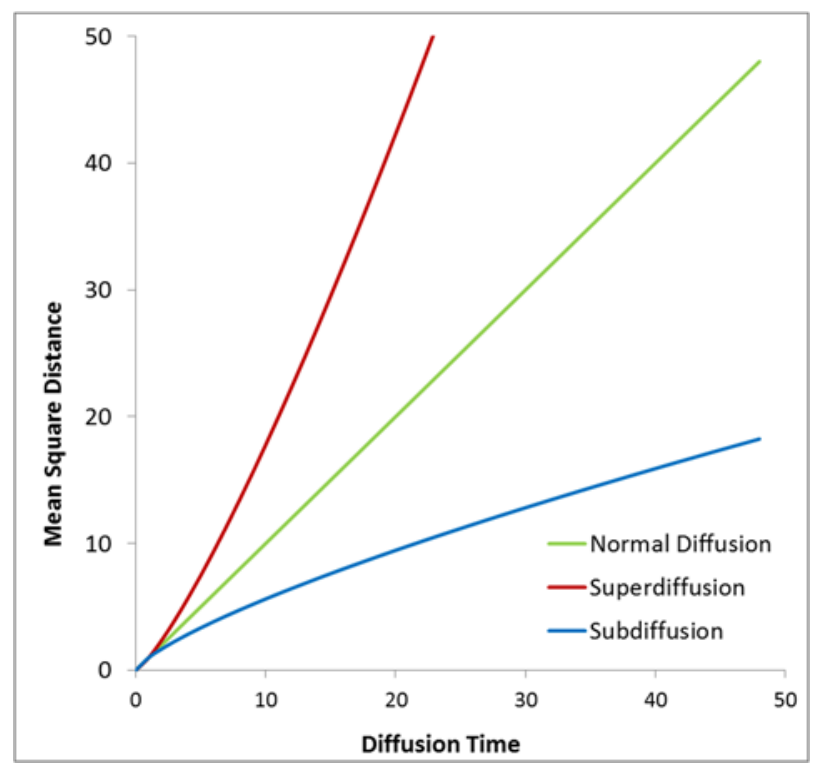

Figure 8. Anomalous diffusion, e.g. super-diffusion (red curve) and sub-diffusion (blue curve), versus conventional or normal diffusion (straight green line). In anomalous diffusion, the mean square distance 
(MSD) of diffusive motion is not proportional to the diffusion time. In case of super-diffusion, the MSD increases faster than linear due to transport processes such as perfusion. In case of sub-diffusion, the MSD increases slower than linear due to barriers such as cellular structure or macro-molecules. In the complex micro environment of liver tissue, there is a mixture of super-diffusion, normal diffusion and sub-diffusion. The relative amount of each type of diffusion process may be altered by pathology while the ADC remains unchanged.

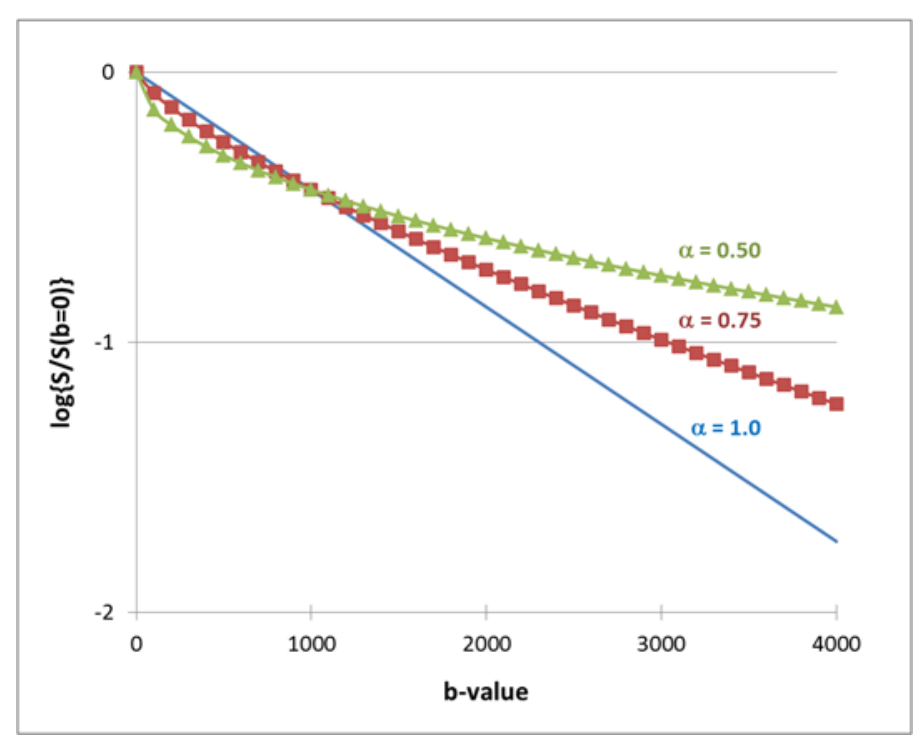

Figure 9. Stretched exponential models of $\alpha=0.75$ and $\alpha=0.5$ versus conventional diffusion model of $\alpha$ $=1.0$. Stretched exponential is one of the models to describe the attenuation of diffusion weighted signal with respected to $b$-value when there is a mixture of super-diffusion, normal diffusion and sub-diffusion. However, the signal attenuation curves of stretched exponential models with different $\alpha$ values can cross each other at a given b-value, which is $1000 \mathrm{~s} / \mathrm{mm}^{2}$ in this example. That means the ADC value would be the same if measured with $b=0$ and $1000 \mathrm{~s} / \mathrm{mm}^{2}$ although the composition of different types of diffusion processes is different. 


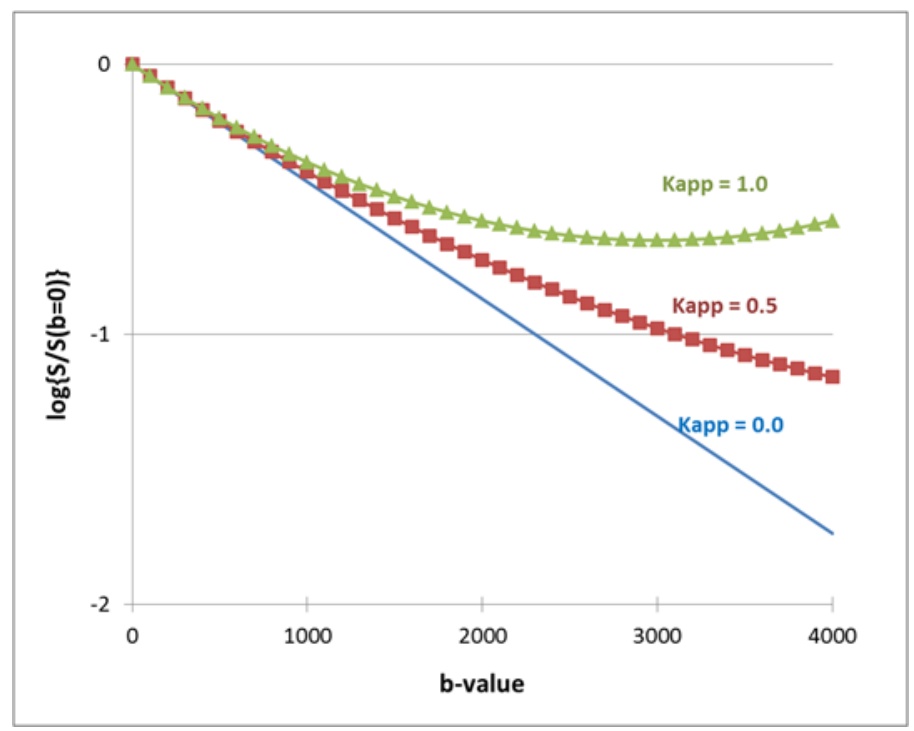

Figure 10. Diffusion kurtosis model of $\mathrm{K}_{\mathrm{app}}=1.0$ and $\mathrm{K}_{\mathrm{app}}=0.5$ versus conventional diffusion model of $\mathrm{K}_{\mathrm{app}}=0$. A deviation of $\mathrm{K}_{\mathrm{app}}$ from zero suggests that the water diffusion is hindered by the tissue structure and a change in $K_{\text {app }}$ is an indication of tissue structural change which could be pathological. Most of the deviation of diffusion kurtosis model from convention diffusion is in the high b-value range, opposite of the IVIM model where most of deviation is in the low b-value range. 